\title{
Impact of Node Mobility with OLSR Protocol
}

\author{
Rakhi Purohit \\ Research Scholar \\ Department of Computer Science \& Engineering \\ Suresh Gyan Vihar University, Jaipur
}

\author{
Bright Keswani \\ Associate Professor \& Head \\ Department of Computer Application \\ Suresh Gyan Vihar University, Jaipur
}

\begin{abstract}
Optimized Link State Routing Protocol has been designed for wireless mobile ad hoc networks. Wireless Mobile Ad Hoc network is consisting of multiple geographically distributed nodes. These network nodes can perform transaction using wireless links and they don't need any fixed infrastructure. In wireless mobile ad hoc network node perform task of host and router both. Because of the nodes have mobility feature so the environment of network is very much dynamic in nature, and the topology of network made frequent. Routing protocol made for this network should be capable to respond to the frequent changes in the network topology. This paper consists of analysis of impact of node mobility with OLSR protocol. This study is performed by simulation of network which has been conducted in famous network simulator NS2.
\end{abstract}

\section{General Terms}

Mobile Ad Hoc Networks, Optimized Link State Routing, Network Simulation, NS2.

\section{Keywords}

OLSR, Routing, Multi Point Relays, Simulation, NS2, Ad Hoc Networks.

\section{INTRODUCTION}

Optimized Link State Routing Protocol generates frequent messages to update the information of the topological of network nodes in network. The nodes in network exchange the information to try to establish a possible route to the destination node in the network. The benefit and plus point of this scheme is that network routes are immediately available at each node to the destination node. It reduces the possible overhead on a particular protocol of network and also used for multipoint relays (MPR). Reducing the time interval for the control transmission of message provides more reactivity in the network topological changes. An OLSR protocol is suitable for large area of networks. The OLSR protocol generally uses two types of control messages namely hello and topology control. This hello messages are utilize to search the information about the link status and the host's neighbors.

\section{OLSR OPERATIONS}

OLSR is a comparatively newer proactive routing protocol. It is an implementation of traditional link- state routing in which each node in network maintains info regarding the network topology. Every node determines the link costs of all the neighbors by broadcasting technique for hello messages in regular intervals. Whenever there is any change in the cost of link, the node broadcasts the information to various other nodes in network. In the classical link-state type of algorithms, this technique is performed by every node by flooding the network with the update packets consist of updated cost of link. Network nodes utilize this information to implement a shortest path algorithm to identify the best network route to a specific destination node in network. OLSR protocol optimizes the link-state protocol in various different ways; it can specify in two ways. In first way, OLSR decrease the size of the update packets which is sent at the time of broadcasts by including only a subset of links to its neighbor node in the network. The links to selected set of neighbor nodes are also known as the multipoint relays (MPR), and the set of various MPRs of a node in network is collection of the minimum set of one node neighbors of that node in network so that this node can reach all of its two hop neighbors by utilizing these network nodes like relay points. Every node in network calculates its set of MPRs by the exchange of information regarding neighborhood with all its neighbor nodes in network. In Second way OLSR does the task somehow differently, means that, instead of each neighbor in network broadcasting the update packets sent from a network node, only the MPR nodes in network will participate in broadcasting of these packets with OLSR protocol. In this way minimizes the traffic of control packets in network during the flooding in network. However, bandwidth saving task achieved by these two techniques and it will be completed on the basis of propagating of incomplete topology information in the network, but the updates include only the nodes in MPR sets and not the node sets of all neighbors node of the broadcasting nodes in network. So that, a shortest path algorithm based on this incomplete topology information will generate routes consist of only the MPR nodes in network. When each node in network has so many neighbor nodes, then OLSR will work very efficient because of the minimum control traffic in the network of nodes.

\section{RELATED WORK}

So many routing protocols proposed by the standard network communities are till now have no agreement on a solution provision due to relevance of wireless or wired networks. The researcher Asma Ahmed [3] proposed discussion on various routing protocols and also comparison between routing protocols also presents the all verity of MANET protocols and prepare a comparative output of the properties of all type of protocol. The researcher Md. Anisur Rahman[6] shows that working differentials can test using changing network load and node mobility also network size. The researcher Samyak Shah [7] checks by comparing the capability of protocols DSDV protocol, AODV protocol and DSR routing protocols with the use of network simulations. The researcher Furqan Haq[9] proposed a comparing of emulated test bed and result of simulation of NS2 simulator and GloMoSim. They use OLSR protocol. The researcher Kapang Lego [12] has done evaluation using simulation for comparison of various performance matrices on various scenarios for manet. In all the papers some of papers discussed the actual simulation results and also changing of found results between various network simulators. On the base of the output of the all research, there is a need for deep and correct planned network simulation and emulation test experiments has been think to required. To identify the 
surety of the output it is necessary to complete detailed simulation.

\section{SIMULATION AND EVALUATION}

\subsection{Simulation Environment}

The simulation model used in this paper is based on network simulator-2, which is used for protocol evaluation in experiment. The instructions of the simulator can be implement to show the topology of the network and to specify the mode of motion of network node, and also to configure the source and the receiver of service, to generate the data trace file [11].

Ns-2 provides good support for network simulation of routing and also multicast routing protocols for wired and wireless networks. NS2 is combination of two simulation tools which are the network simulator (ns) which contains various commonly used IP protocols and the network animator (nam) tool is utilize to actually visualize the network simulations.

\subsection{Traffic Model}

In this work CBR has used for continuous bit rate traffic sources for network simulation. The network made by source and destination nodes commonly distributed as randomly and also 512-byte data packets is used for analysis of task in experiment. The pairs count made for source and destination and also the sending rate of packet of node pairs are time to time changed to varying the load proposed for the network.

\subsection{Mobility Model}

The mobility model [1][10] is the random waypoint model in a specified area of rectangular field which is configurations as 500 multiply be 500 area with the 100 nodes. In network communication every data packet starts its route from a specific random source location to a random node destination by a specified and randomly communication network speed. When it is reached to destination, then again another random node destination is specified. In the network simulation time is varied which responsible to effects the relative speeds of the nodes in network. Node mobility in network is same and traffic scenarios which are used by the protocols to get good output of experiments.

\subsection{Simulation Model}

Network Simulator NS2 [7], is an effective and simple event driven simulation software which is useful in testing of the implementation of logically networks and also make shorten the burden of actual network implementation. NS2 provides various capabilities to effective simulation of wired and wireless network and also support most of protocols. Here a simulation have performed to evaluate the performance of Mobile Ad Hoc Network routing protocols OLSR based network throughput with various parameters[10][11].

Radio Model - Two Way Ground

Traffic Source -CBR

Packet Size - 512 Bytes

Network Speed - $10 \mathrm{~m} / \mathrm{s}$

Area - 500X500

Number of Nodes 100

MAC - Mac/802_11

Simulation Time $20,40,60,80 \& 100$
To achieve this goal and aim it actually need to investigate that how OLSR protocol performs when the load of nodes increases with the mobility of network nodes. In this work the simulation has been done by network simulator 2.34 .

\section{PERFORMANCE MATRICES}

Some important matrices have evaluated for the analysis of routing protocol in different network scenario and different experimental setups in well-known network simulator software. [11].

\subsection{Average Throughput}

The average throughput is performance metrics which is a ratio of the total amount of data reaches to a specific receiver node from a sender node to the time which it takes. In the specific communication networks the network throughput is the average rate of a perfect message delivery in a particular transmission channel in simulated network. This data may be passed on a particular physical link or can be the logical link, or it can be pass through the specific particular network node in simulated network. The network throughput is generally calculated in the form of bits per second.

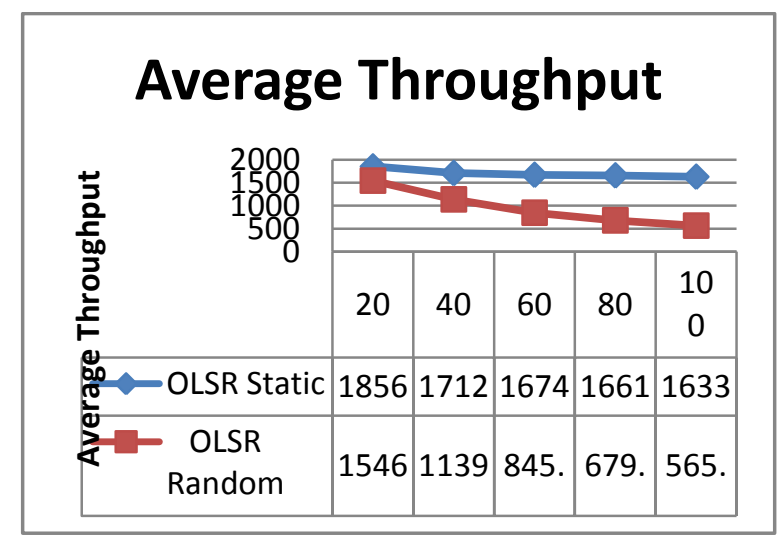

Figure 1 : Average Throughput

Based on simulation results, the throughput value of network based on static vector nodes and OLSR based network in different simulation time initially higher as 1856.32 but it decreases as simulation time increases as on 100 simulation time it reach on 1632.56 whereas, the throughput value of network based on mobile random nodes and OLSR based network in different simulation time initially 1546.35 but it decrease as simulation time increase as on 100 simulation time it reach on 565.18 which shows that in mobility environment OLSR based network cannot provide good network throughput in mobility and more simulation time.

\subsection{Packet Delivery Ratio}

In network the packet delivery ratio is the ratio between lot of received packets by the destination node and the number of the data packets sent by the source node in network. It is the ratio between the number of packets actually transmitted by a network traffic source and the number of packets received by a network traffic sink. Packet delivery ratio can measures the loss rate as seen by transport protocols and as like, it characterizes the correctness and the efficiency of ad hoc network routing protocols. Packet delivering at high rate ratio is very much necessary in any of the network. The packet ratio of the originated applications of the data packets of each of the protocol is able to deliver at varying time in network. Packet delivery ratio is understood by the ratio of the number of delivered data packet to the destination. It 
illustrates level of the delivered data to the destination node in network.

$\sum$ Number of packet receive / $\sum$ Number of packet send

\section{Packet Delivery Ratio}

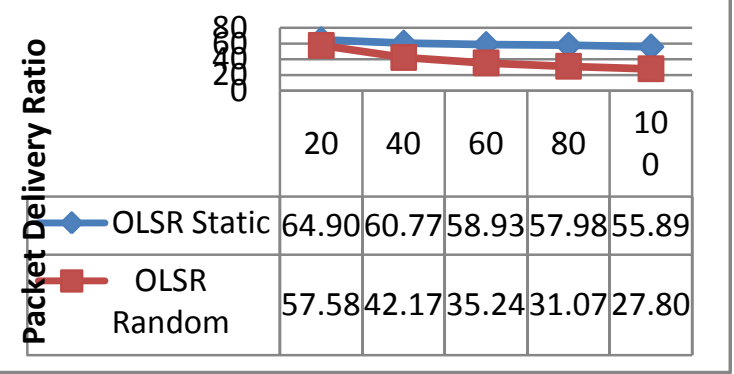

Figure 2 : Packet Delivery Ratio

Based on simulation results, the packet delivery ratio of network based on static vector nodes and OLSR based network in different simulation time initially higher as 64.9028 but it decreases as simulation time increases as on 100 simulation time it reach on 55.8923 whereas, the packet delivery ratio of network based on mobile random nodes and OLSR based network in different simulation time initially 57.5796, but it decrease as simulation time increase as on 100 simulation time and it reach on 27.8042. Shows that OLSR based network can provide good packet delivery ratio in static random nodes based network.

\subsection{Average End-to-End delay}

Average End to End Delay is the time which is elapsed when a data packet is sent from the source node in network and also it is received by the desired destination node in network. It includes delays as delay for route discovery, propagation time, time to transfer data, and intermediate queuing delays. It is the average time taken by a data packet to travel from source to destination. Also it includes the delay caused by the network route discovery process and the queue in data packet transmission from sender to receiver. Only the data packets which are successfully received to destinations will be counted.

$\sum$ (arrive time - send time) $/ \sum$ Number of connections

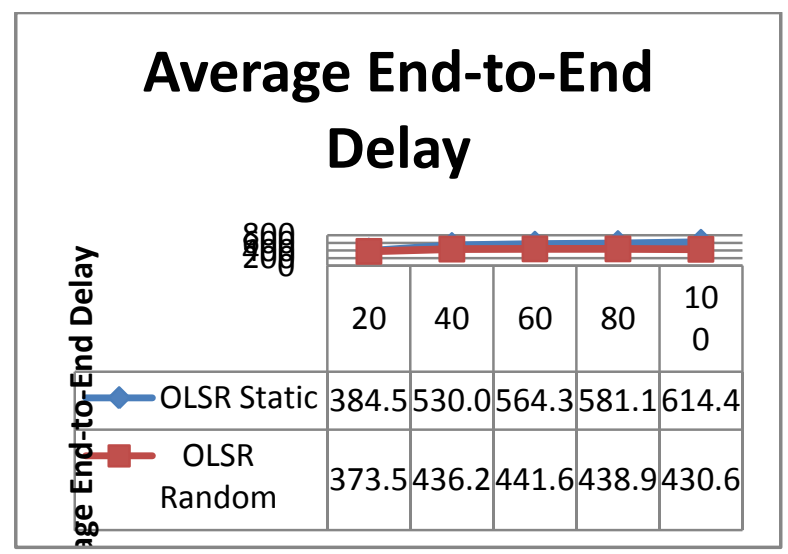

Figure 3 : Average End to End Delay

Based on simulation results, the average end to end delay of network based on static vector nodes and OLSR based network in different simulation time initially low as $\mathbf{3 8 4 . 5 2 5}$ but it increases as simulation time increases as on 100 simulation time it reach on 614.461 whereas, the end to end delay of network based on mobile random nodes and OLSR based network in different simulation time initially 373.552, but it increase as simulation time increase as on 100 simulation time and it reach on 430.674. It shows that OLSR based network can provide good end to end delay in static random nodes based network.

\subsection{Dropped Packet}

Dropped packet is the amount of packets which are generated by transmitting node but not received by receiver node and lost in network due to some specific reason, the formula to calculate this parameter need packet sending and packet received. The number of packets that generated but not received so dropped during the network simulation.

Dropped Packet $=$ Number of packet send - Number of packet received.

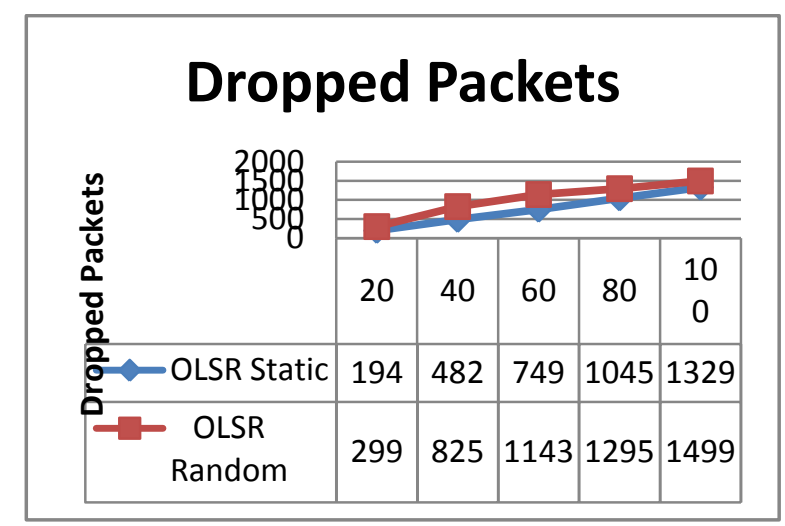

Figure 4 : Dropped Packets

Based on simulation results, the dropped packets of network based on static vector nodes and OLSR based network in different simulation time initially low as 194 but it increases as simulation time increases as on 100 simulation time it reach on 1329 whereas, the dropped packets of network based on mobile random nodes and OLSR based network in different simulation time initially 299 , but it increase as simulation time increase as on 100 simulation time and it reach on 1499. It show that OLSR based network have more packet loss in random nodes based network.

\section{CONCLUSION}

It has been concluded that by different simulation and evaluation that the OLSR provide good throughput and packet delivery ratio with static nodes in network whereas mobility in network effect on throughput and packet delivery ratio and make it low due to varying topology due to node mobility. With static node in OLSR average end to end delay is initially similar to node with mobility but as simulation time increases it reduce with random mobile node whereas increase in static nodes so mobility in node effect protocol behavior in some aspects.

\section{FUTURE WORK}

In the future, like to perform extensive complex simulations to gain a more in-depth analysis of the wireless mobile ad hoc routing protocols. Also try to enhance some new feature in protocol. Also like to introduce new protocol, not only for the various routing protocols but for more and more vast areas of wireless networking and various important concepts in computer network and communications. 


\section{ACKNOWLEDGMENT}

There is a special thanks to the expert persons who helped a lot to setup proper simulation environment so that it was possible to do experiment and analyze different scenarios and conclude better results.

\section{REFERENCES}

[1] Xi Hu , Jinkuan Wang, Cuirong Wang “ Mobilityadaptive Routing for Stable Transmission in Mobile Ad Hoc Networks “JOURNAL OF COMMUNICATIONS, VOL. 6, NO. 1, February 2011

[2] Rakesh Poonia, Amit Kumar Sanghi, Dr. Dharm Singh "DSR Routing Protocol in Wireless Ad-hoc Networks: Drop Analysis " International Journal of Computer Applications (0975 - 8887) Volume 14- No.7, February 2011

[3] Asma Ahmed, A. Hanan, Shukor A. R., Izzeldin M. "Routing in Mobile Ad hoc Network " IJCSNS International Journal of Computer Science and Network Security, VOL.11 No.8, August 2011

[4] Beigh Bilal Maqbool Prof.M.A.Peer "Classification of Current Routing Protocols for Ad Hoc Networks - A Review " International Journal of Computer Applications (0975 - 8887) Volume 7- No.8, October 2010

[5] Sunil Taneja , Ashwani Kush "A Survey of Routing Protocols in Mobile Ad Hoc Networks" International Journal of Innovation, Management and Technology(2010 - 0248) Vol. 1, No. 3,August 2010

[6] Md. Anisur Rahman, Md. Shohidul Islam, Alex Talevski "Performance Measurement of Various
Routing Protocols in Ad-hoc Network " Proceedings of the International MultiConference of Engineers and Computer Scientists 2009 Vol I ,IMECS 2009, March 2009

[7] Samyak Shah, Amit Khandre, Mahesh Shirole, Girish Bhole " Performance Evaluation of Ad Hoc Routing Protocols Using NS2 Simulation " Mobile and Pervasive Computing (CoMPC-2008)

[8] Padmini Misra "Routing Protocols for Ad Hoc Mobile Wireless Networks" "http://www.cis.ohio-state.edu/ misra"

[9] Furqan Haq and Thomas Kunz "Simulation vs. Emulation: Evaluating Mobile Ad Hoc Network Routing Protocols "Systems and Computer Engineering Carleton University Ottawa, Ont., Canada K1S 5B

[10] Gianni A. Di Caro ," Analysis of simulation environments for mobile ad hoc networks " Technical Report No. IDSIA-24-03 IDSIA / USI-SUPSI , Dalle Molle Institute for Artificial Intelligence Galleria, Switzerland , December 2003

[11] Karthik sadasivam " Tutorial for Simulation-based Performance Analysis of MANET Routing Protocols in ns-2 “

[12] KAPANG LEGO ， PRANAV KUMAR SINGH, DIPANKAR SUTRADHAR "Comparative Study of Adhoc Routing Protocol AODV, DSR and DSDV in Mobile Adhoc Network" Indian Journal of Computer Science and Engineering Vol.1 No.4 364-371 